\title{
Predictive serum markers for severe COVID-19 illness
}

\author{
Masaya SUGIYAMA ${ }^{1 *}$ \\ 1 National Center for Global Health and Medicine, 1-7-1 Kohnodai, Ichikawa, Chiba 272-8516, Japan
}

Key words: COVID-19, severe illness, predictive markers, cytokine, chemokine

\begin{abstract}
Introduction
A pandemic caused by a new coronavirus (severe acute respiratory syndrome coronavirus 2 (SARS-CoV-2)) has emerged, and a large number of infections and deaths are being reported daily worldwide. Vaccines against SARS-CoV-2 infection have been developed and are being administered globally, with the hope that vaccination will reduce the number of infections and deaths.

Most patients with COVID-19 infection have mild symptoms and recover from the disease, but some develop more severe symptoms (Fig. 1) [1]. In the early stages of COVID-19 outbreak, biochemical tests and other assays were used to search for markers that could predict which patients would develop severe disease, and Interleukin-6 (IL-6) was reported as a candidate for prognostication.

Obesity, chronic heart disease, and chronic kidney disease have been identified as risk factors for the transition to moderate II or higher COVID-19 symptom, and many reports have been published worldwide. Lactate dehydrogenase (LDH), C-reactive protein (CRP), and IL-6 have been reported as predictive markers for severe disease [2, 3]. However, in terms of disease uniqueness, sensitivity, and specificity, it has been difficult to obtain a positive diagnosis rate that is suitable for clinical applications.
\end{abstract}

\section{Novel predictive Markers for Severe COVID-19 IIIness}

Our center, National Center for Global Health and Medicine, has been accepting COVID-19 patients since the early days of the pandemic, and we have been storing blood samples collected from patients over time. With an aim to predict which patients would progress to moderate II or higher COVID-19, we searched for appropriate diagnostic markers since the beginning of 2020. We conducted a comprehensive analysis of liquid components in the blood to identify the factors associated with changes in the disease state. By examining approximately 70 factors, mainly cytokines and chemokines, we identified factors in the blood that could be used to identify patients likely to become severely ill, before they developed moderate II or higher COVID-19. These factors were interferon lambda 3 (IFN- $\lambda 3$ ), C-X-C motif ligand (CXCL) 9, CXCL10, IL-6, and C-C motif ligand (CCL) 17 [4]. Of these, CXCL9, CXCL10, and IL-6 were reported in previous studies, and these results were replicated; however, IFN- $\lambda 3$ and CCL17 were identified as novel prognostic factors. Currently, these two biomarkers have been approved for predicting moderate II or higher COVID-19 and are the focus of this review.

\section{Usefulness of Serum CCL17 Test in an Early Phase of COVID-19}

Even when tested in the early stages of mild SARS-CoV-2 infection, the serum levels of CCL17 differed between patients who recovered from mild disease and those who later became severely ill (Fig. 2A). Patients with severe disease showed lower levels of CCL17 than healthy subjects, even during the initial mild phase of infection, and remained low until the severe phase. In contrast, patients with mild disease had values similar to those of healthy subjects. When we checked the CCL17 values in patients with various chronic diseases, we found that CCL17 was low only in patients with severe COVID-19.

\section{Usefulness of Serum IFN- $\lambda 3$ Test in COVID-19}

We also analyzed the changes in IFN- $\lambda 3$ levels in patients with COVID-19. The results indicated that patients who progressed from mild to moderate disease II showed a characteristic peak value a few days before progression (Fig. 2B). The concentration of IFN- $\lambda 3$ rapidly increased and then decreased rapidly. During the decrease, the patients developed moderate II disease, which causes increased oxygen demand. Higher values at this peak tended to indicate more severe symptoms. This point should be clarified by the accumulation and analysis of data in the future. The peak value did not remain high, but only transiently increased.

In contrast, patients who recovered from mild illnesses rarely showed the characteristic peak changes described above, and the values remained low. 

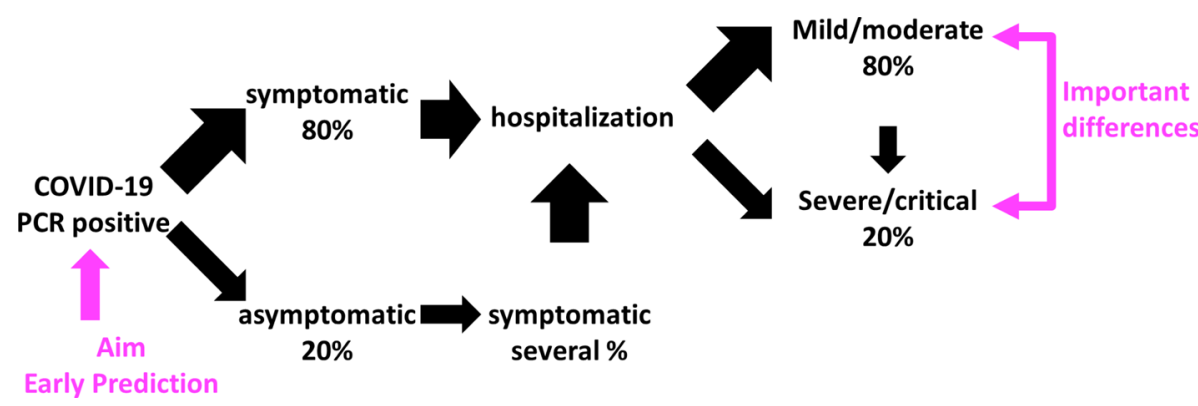

$20 \%$

Fig. 1. Schematic flowchart demonstrating the events after severe acute respiratory syndrome coronavirus 2 (SARS-CoV-2) infection.

\section{A Mild/moderate}

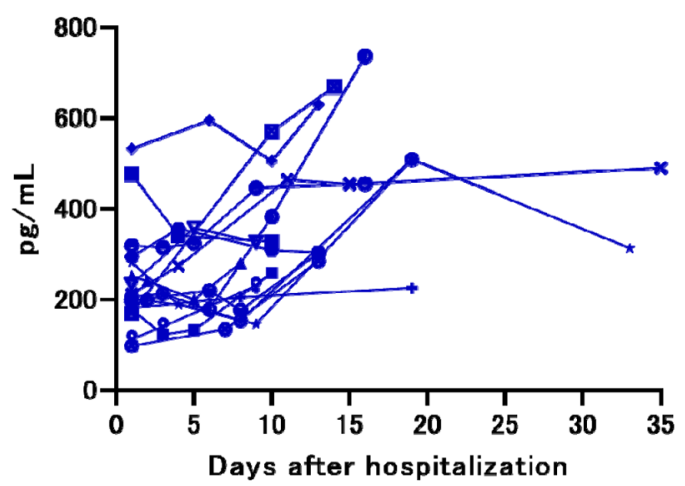

B

Mild/moderate

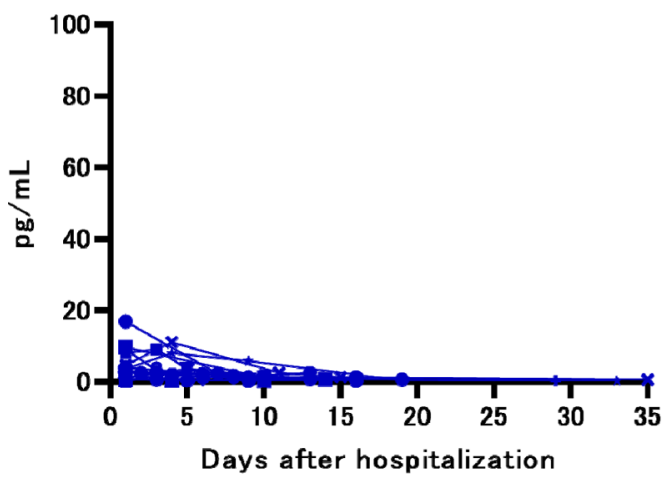

\section{CCL17}

\section{Severe/critical}

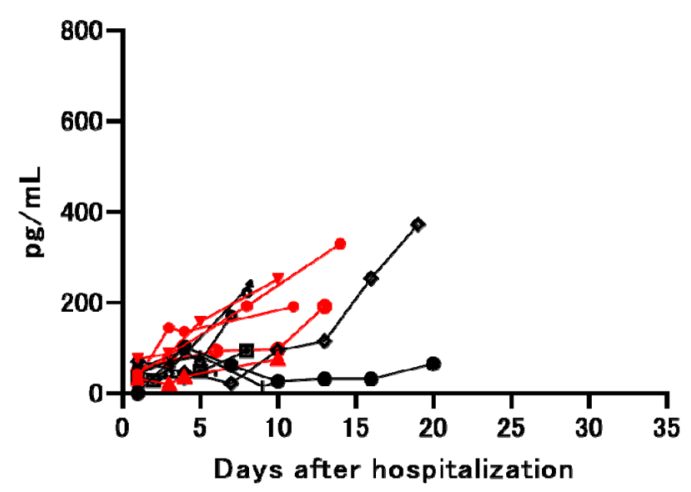

$\underline{\text { IFN- }-\lambda 3}$

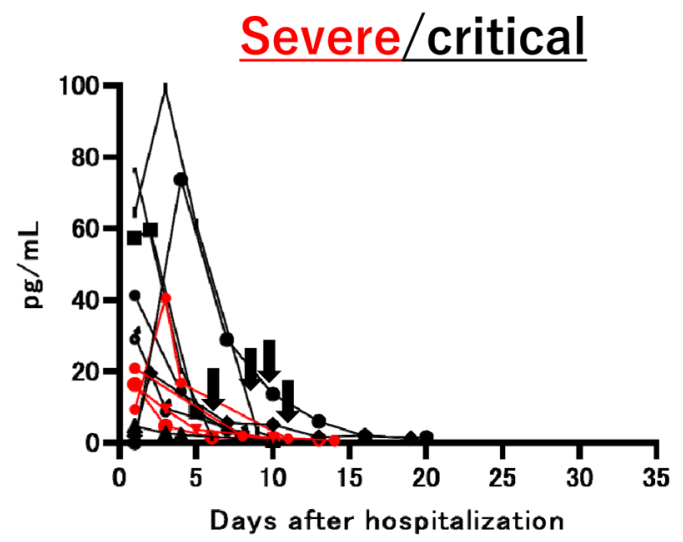

Fig. 2. Dynamics of predictive markers in the serum of COVID-19 patients. Serum concentrations of A) C-C motif ligand (CCL) 17 and B) interferon lambda 3 (IFN- $\lambda 3$ ) from the day of admission till the end of hospital stay. Blue line: mild/moderate patients, red line: severe patients, black line: critical patients. The black arrow in representative patients indicates the point of development of severe illness. After the peak in IFN- $\lambda 3$, severe illness developed in each patient (Fig. 2B, right).

Differences between IFN- $\lambda 3$ and IL- 6 tests in COVID-19 Severe IIIness

As reported previously, IL-6 has been reported to be associated with moderate II and severe disease since the early stages of the COVID-19 outbreak; an IL-6 test was approved in Japan prior to the IFN- $\lambda 3$ test, but caution is required because it differs from IFN- $\lambda 3$ in its indications and target disease states.

The indications for the IL- 6 test have been established as "to assist in the determination of the severity of illness in patients who require emergency transport, intensive care, or are under intensive care management" In other words, it is used to examine the severity of symptoms in patients 
with moderate II or higher COVID-19. Because the test is not limited to COVID-19, it can be used for a wide range of diseases with a systemic inflammatory response.

In contrast, the IFN- $\lambda 3$ test is available only for COVID-19; it is performed at the mild or moderate I stage of COVID-19 to predict the risk of patients progressing to moderate II or higher stages. Based on the characteristic rapid increase in value, as shown in Fig. 2B, that occurs before the onset of moderate II stage, multiple measurements can be taken until the test result is above the reference levels. If the test result value is above the reference level, it indicates that the patient is likely to progress to moderate II or higher stages a few days later.

\section{Conclusions}

Both the CCL17 and IFN- $\lambda 3$ tests were conducted using an automatic testing device provided by the manufacturer, and results were obtained within an hour. Because the devices themselves are installed in many laboratories of major testing companies and large facilities, such as university hospitals, these laboratories can be used in setting up a testing system.

In COVID-19, whether the disease progresses to moderate II or higher is an important question in clinical practice. At present, a considerable knowledge of treatment methods for COVID-19 has accumulated. The probability of death is decreasing, even in patients with moderate II or higher disease, as long as appropriate treatment is received. It has also been found that early therapeutic interventions can lead to a shorter recovery period.

However, because it is not possible to treat all COVID-19 patients with medication, it is necessary to distinguish the patients who require therapeutic intervention from those who do not. Because the CCL17 and IFN- $\lambda 3$ tests require blood sampling, it is necessary to develop simpler tests to aid in clinical diagnosis. It is hoped that COVID-19 treatment can be implemented efficiently through the appropriate use of diagnostic markers.

\section{Conflict of Interest}

The authors declare no conflicts of interest associated with this manuscript.

\section{Funding}

This study was supported by grants (JP19fk0108164, JP19fk0108104, and JP20fk0108104) from the Japan Agency for Medical Research and Development (AMED).

\section{Acknowledgements}

The authors would like to thank the patients and their families for participating in this study and the medical staff at the National Center for Global Health and Medicine for their support. In addition, we appreciate the support of those who participated in the Biobank and the NCMG Biobank.

\section{References}

1. Kutsuna, S. 2020. Coronavirus disease 2019 (COVID-19): research progress and clinical practice. Glob Health Med 2: 78-88. [Medline] [CrossRef]

2. Cummings, M. J., Baldwin, M. R., Abrams, D., Jacobson, S. D., Meyer, B. J., Balough, E. M., Aaron, J. G., Claassen, J., Rabbani, L. E., Hastie, J., Hochman, B. R., Salazar-Schicchi, J., Yip, N. H., Brodie, D. and O'Donnell, M. R. 2020. Epidemiology, clinical course, and outcomes of critically ill adults with COVID-19 in New York City: a prospective cohort study. Lancet 395: 1763-1770. [Medline] [CrossRef]

3. Yang, Y., Shen, C., Li, J.,Yuan, J., Wei, J., Huang, F., Wang, F., Li, G., Li, Y., Xing, L., Peng, L., Yang, M., Cao, M., Zheng, H.,Wu, W., Zou, R., Li, D., Xu, Z., Wang, H., Zhang, M., Zhang, Z., Gao, G.F., Jiang, C., Liu, L., Liu, Y. 2020. Plasma IP-10 and MCP-3 levels are highly associated with disease severity and predict the progression of COVID-19. J. Allergy Clin. Immunol. 146: 119-127e4. [Medline] [CrossRef]

4. Sugiyama, M., Kinoshita, N., Ide, S., Nomoto, H., Nakamoto, T., Saito, S., Ishikane, M., Kutsuna, S., Hayakawa, K., Hashimoto, M., Suzuki, M., Izumi, S., Hojo, M., Tsuchiya, K., Gatanaga, H., Takasaki, J., Usami, M., Kano, T., Yanai, H., Nishida, N., Kanto, T., Sugiyama, H., Ohmagari, N. and Mizokami, M. 2021. Serum CCL17 level becomes a predictive marker to distinguish between mild/moderate and severe/ critical disease in patients with COVID-19. Gene 766: 145145. [Medline] [CrossRef] 